\title{
Contaminación atmosférica y daño cardiovascular
}

\author{
O scar Román A ${ }^{1}$, María José Prieto ${ }^{2 a}$ y Pedro Mancilla2a. \\ Atmospheric pollution and \\ cardiovascular damage
}

The damaging effect of atmospheric pollution with particulate matter and toxic gases on the respiratory system and its effect in the incidence and severity of respiratory diseases, is well known. A similar effect on the cardiovascular system is currently under investigation. Epidemiological studies have demonstrated that the inhalation of particulate matter can increase cardiovascular disease incidence and mortality, specially ischemic heart disease. The damage would be mediated by alterations in the autonomic nervous system, inflammation, infections and free radicals. In human studies, environmental pollution is associated with alterations in cardiac frequency variability and blood pressure and with changes in ventricular repolarization. Experimentally, an enhancement of ischemia, due to coronary obstruction, has been demonstrated. The study of the toxic effects of environmental pollution over the cardiovascualr system, is an open field, specially in Chile, were the big cities have serious contamination problems (Rev Méd Chile 2004; 132: 761-7).

(Key Words: Air pollution; Cardiovacular diseases; Coronary disease)

Recibido el 2 de julio, 2003. Aceptado en versión corregida el 1 de abril, 2004.

${ }^{1}$ Departamento de Medicina, Campus Centro, Universidad de Chile. ${ }^{2}$ Ministerio de Salud, Unidad de Salud Respiratoria.

akinesiólogo.

$\mathrm{D}$ esde hace aproximadamente 15 años se ha acumulado un número creciente de evidencias epidemiológicas, experimentales y clínicas respecto a la relación entre la concentración de material particulado atmosférico (PM) y diversos daños en los sistemas respiratorio $\mathrm{y}$

Correspondencia a: Dr. Oscar Román A. Mac Iver 544. Santiago de Chile. Fono: 6300433.

E-mail: oscarromanalemany@hotmail.com cardiovascular $^{1,2}$. En un comienzo, el interés de las investigaciones se centró en el daño respiratorio, atendiendo a los crecientes niveles de contaminación atmosférica, producidos por la vida moderna en las grandes ciudades y a su relación con la incidencia de enfermedades respiratorias $^{3,4}$. En nuestro país, en 1989, Aranda $\mathrm{y} \mathrm{col}^{5}$ reconocían una correlación entre la climatología de la ciudad de Santiago y su efecto sobre la contaminación atmosférica y la salud de la población. Los mismos autores, en 1991, comunicaron una relación entre la conta- 
minación atmosférica por partículas grandes $\left(\mathrm{PM}_{10}\right)$ y la mortalidad por neumonía en niños de Santiago ${ }^{6}$. Posteriormente Ostro y $\mathrm{col}^{7} \mathrm{y}$ Sanhueza y $\mathrm{col}^{8}$, también en Santiago, encontraron una importante correlación entre la concentración de partículas grandes $\left(\mathrm{PM}_{10}\right)$ y la mortalidad debida tanto a enfermedades respiratorias como cardiovasculares. Sin embargo, en el mismo año, Avendaño y col ${ }^{9}$ no observaron correlación entre la concentración de $\mathrm{PM}_{10}$ con un aumento de la demanda de hospitalizaciones en lactantes y niños, por lo que postularon un rol adicional de virus respiratorio y de partículas más pequeñas $\left(\mathrm{PM}_{2,5}\right)$.

En cuanto al sistema cardiovascular (SC-V), las investigaciones son más recientes, pero también significativas, respecto a la relación de la contaminación atmosférica con la mortalidad y morbilidad de algunas patologías, especialmente angina de pecho, arritmias, infarto miocárdico y accidente cerebrovascular ${ }^{1,2}$. En nuestro medio, Sanhueza y $\mathrm{col}^{8}$ han estimado en 183/año el riesgo de muertes por enfermedad cardiovascular (C-V) debidas a $\mathrm{PM}_{10}$ y en 343/año las debidas a $\mathrm{PM}_{2,5}$ en personas mayores de 65 años.

Esta revisión tiene como objetivo describir los estudios epidemiológicos, experimentales y clínicos que apoyan la existencia de un efecto nocivo de la contaminación atmosférica sobre el SC-V y analizar las hipótesis patogenéticas planteadas.

\section{ESTUDIOS EPIDEMIOLÓGICOS}

Se ha observado mayor frecuencia de hospitalizaciones y mayor mortalidad por enfermedades $\mathrm{C}-\mathrm{V}$ en los períodos de mayor contaminación atmosférica $^{2}$. En Santiago, se ha demostrado también mayor riesgo de muertes $\mathrm{C}$ - $\mathrm{V}$ en relación a las concentraciones de PM, siendo mayor el riesgo de la exposición a material particulado fino $\left(\mathrm{PM}_{2,5}\right)$, especialmente en las personas mayores de 65 años $^{5}$. Estudios epidemiológicos recientes han descartado una simple asociación 0 relación falsa entre los períodos de mayor concentración de partículas ambientales y el incremento de muertes y hospitalizaciones por episodios isquémicos ${ }^{8}$.
Recientemente, en diabéticos (pacientes altamente sensibles a las afecciones $\mathrm{C}-\mathrm{V}$ ) expuestos a incrementos del PM, se ha observado que el riesgo de hospitalización por enfermedad cardíaca ha sido el doble que en la población general ${ }^{10} \mathrm{y}$ que además, se incrementa también la mortalidad asociada ${ }^{11}$.

Se ha observado que los individuos portadores de afecciones crónicas, especialmente pulmonares, tienen también mayor riesgo de hospitalizaciones por enfermedad $\mathrm{C}-\mathrm{V}$ cuando están expuestos a contaminación atmosféri$\mathrm{ca}^{12}$.

\section{ESTUDIOS EXPERIMENTALES}

En roedores y perros, se ha podido establecer que la inhalación de PM determina liberación de mediadores de inflamación en el epitelio pulmonar, los que al pasar a la sangre, provocan inflamación en las arteriolas ${ }^{13}$. En otro estudio, utilizando un modelo de ateroesclerosis en lauchas genéticamente deficientes en apoliproteína E (por disrupción del gene ApoE, que lleva a una hipercolesterolemia espontánea, con lesiones en los vasos que se parecen a las lesiones ateromatosas humanas observadas a los 3 a 5 años de edad), se demostró que tanto las lauchas deficientes en Apo E como las controles, expuestas a PM de $125 \mathrm{um} / \mathrm{ml}^{3}$ obtenido en la ciudad de Washington, presentaron una reducción de la frecuencia cardíaca, pero las Apo E deficientes mostraron además una significativa tendencia al aumento de la presión arterial y de la variabilidad de la frecuencia cardíaca ${ }^{14}$.

En otra experiencia, ratas expuestas a PM en concentraciones de 160 a $200 \mathrm{ug} / \mathrm{m}^{3}$ por períodos de $4 \mathrm{~h}$, alternados con períodos similares de aire filtrado, mostraron un aumento significativo de arritmias supraventriculares en los períodos de exposición al $\mathrm{PM}^{15}$.

Willenius y $\mathrm{col}^{16,17}$ provocaron isquemia ocluyendo la coronaria anterior de perros conscientes, a los que se exponía a PM por $6 \mathrm{~h}$ al día, durante 4 días seguidos. Inmediatamente después de cada exposición, se observó aumento de la elevación de ST, aunque no hubo variación de la frecuencia 
cardiaca (FC) ni de la incidencia de arritmias. Concluyeron que el PM aumenta la isquemia aguda miocárdica probablemente por cambios fisiopatológicos en la respuesta vascular.

\section{ESTUDIOS CLINICOS}

Los estudios en el hombre se han centrado en los efectos del PM en la variabilidad de la frecuencia cardíaca y en los cambios de la repolarización en el electrocardiograma (ECG). Se ha observado que sujetos normales, expuestos en forma intermitente y alternada, durante $2 \mathrm{~h}$, a inhalación de aire y de partículas de carbón de 10 a $25 \mathrm{ug} / \mathrm{m}^{3}$ durante ejercicio, mostraban en el registro continuo del ECG una respuesta parasimpática atenuada post-ejercicio durante la exposición a las partículas ultra finas. Esta respuesta vagal disminuida no perduraba más allá de $3 \mathrm{~h}^{18}$. Además, en esa misma experiencia, se demostró que las partículas ultrafinas alteraban la repolarización ventricular, como se pudo evidenciar por el estudio del intervalo QT corregido. El aumento del QT inducido por el ejercicio inhalando aire era atenuado con la exposición al PM y el efecto persistía por lo menos $21 \mathrm{~h}$ después. Por tanto, la modificación del QT (corregido) no se explicaba por cambio de la frecuencia cardíaca18,19. Es posible que los cambios de la repolarización ventricular se puedan deber a un efecto indirecto, vía sistema nervioso autónomo o a un efecto directo sobre el intercambio iónico en el miocardio ventricular, por mecanismos aún no precisados ${ }^{19,20}$. Además, estos cambios podrían influir en la aparición de paro cardiorrespiratorio en personas con y sin enfermedad cardíaca reconocida $^{21}$.

Los estudios clínicos en el hombre se han extendido a las implicancias del efecto del PM en los pacientes con enfermedad cardíaca preexistente, relacionándolo con la variabilidad de la FC, de la repolarización y de los trastornos enzimáticos y proteicos estudiados clásicamente en la enfermedad isquémica ${ }^{22}$. En un estudio en ciudad de México, en adultos mayores se observó que ambientes con $\mathrm{PM}_{2,5}$ y ozono podían reducir la variabilidad de la FC y que tal efecto era mayor en los pacientes hipertensos ${ }^{23}$. En
Taiwán se ha observado que el riesgo de accidente cerebro vascular (isquémico y hemorrágico) es mayor en los días de aumento del $\mathrm{PM}_{10} \mathrm{y}$ de $\mathrm{NO}_{2}{ }^{24}$.

\section{MECANISMOS PATOGENÉTICOS}

Se han estudiado diversos mecanismos biológicos para explicar la asociación significativa entre concentración de material particulado atmosférico y exceso de mortalidad y morbilidad $\mathrm{C}-\mathrm{V}$. Tales mecanismos incluyen la alteración del sistema nervioso autónomo, la inflamación, la inmunidad, los radicales libres y el daño endotelial.

El material contaminante lo constituyen las partículas en suspensión y los gases tóxicos. El material particulado (PM) se clasifica en el de partículas finas, de menos de 2,5 micrones $\left(\mathrm{PM}_{2,5}\right)$ y mayores, de $10\left(\mathrm{PM}_{10}\right)$, siendo las pequeñas las que se asocian más fuertemente al exceso de mortalidad. Los gases tóxicos son el ozono $\left(\mathrm{O}_{3}\right)$, $\mathrm{SO}_{2}, \mathrm{NO}_{2}, \mathrm{CO}_{2}$ y otros compuestos orgánicos volátiles ${ }^{1}$.

En base, principalmente, a los estudios experimentales, los investigadores han planteado una hipótesis integradora de los mecanismos patogenéticos involucrados ${ }^{1}$. Según ella, la puerta de entrada del material particulado es el pulmón, en cuyo interior, la interacción del PM con el epitelio respiratorio determina una serie de efectos, entre los que destacan cambios en el tono autonómico, inflamación, acción de citoquinas, aumento de los radicales libres o sustancias reactivas al $\mathrm{O}_{2}$ (denominadas también substancias ROS o reactive oxygen species), la alergia y la infección respiratoria. Estos cambios provocarían diversos efectos a distancia, como activación leucocitaria en la sangre, alteraciones de la coagulación, cambios en la concentración del $\mathrm{O}_{2}$ y del mecanismo ácido-base y, en forma destacada, disfunción endotelial a nivel del sistema C-V (Figura 1). Tales cambios se pueden deber tanto al material particulado como a sus productos de reacción.

A. Sistema nervioso autónomo. La puerta de entrada del MP es el pulmón, donde la interac- 


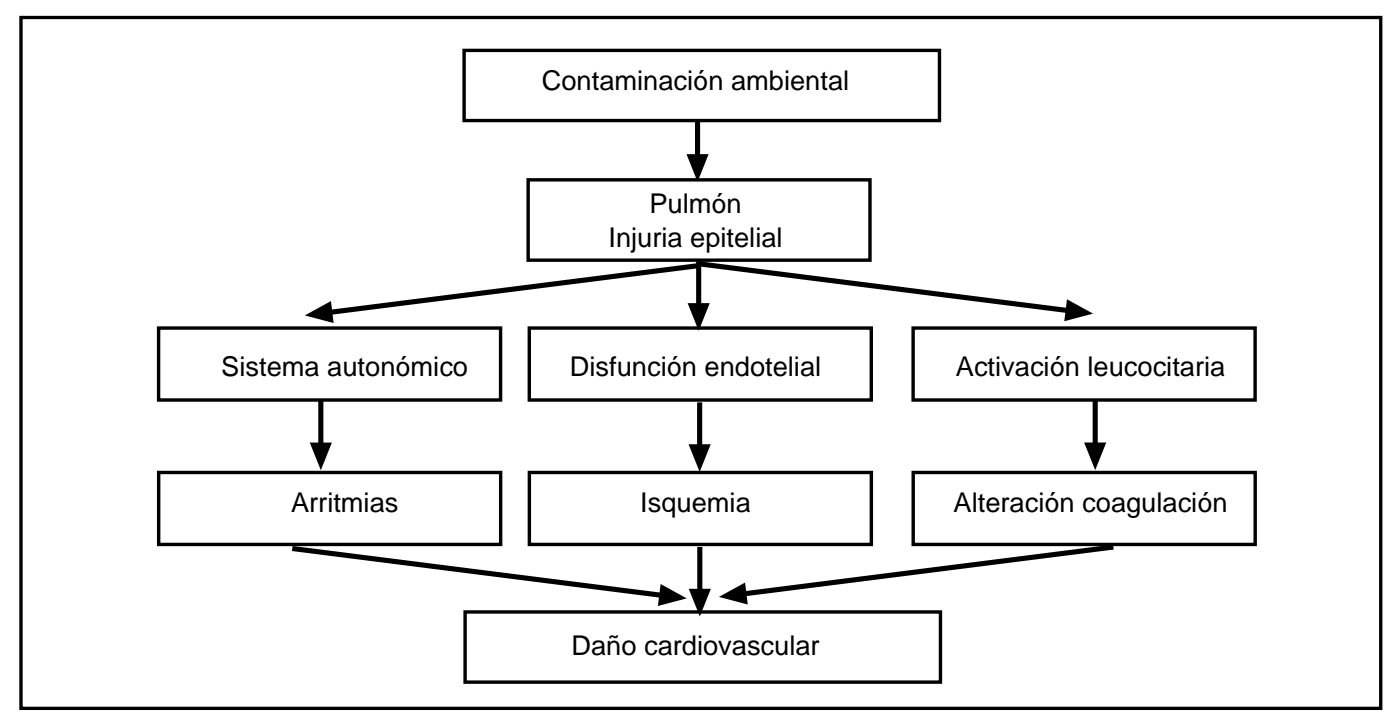

FiguRA 1. Hipótesis integradora de los mecanismos patogenéticos.

ción de las partículas con el epitelio produce un efecto de estimulación de los nervios sensitivos, que lleva a una disfunción del sistema autonómico caracterizada por reducción de la frecuencia cardíaca, disminución de su variabilidad, especialmente en sus componentes de alta frecuencia y reducción de la presión arterial. Estos cambios han sido observados tanto experimentalmente como en seres humanos ${ }^{25,23}$ y se ha establecido la hipótesis que podrían ser provocados mediante una acción indirecta, vía sistema autonómico o bien, en forma directa, alterando la función de los canales iónicos en el miocardio mismo. La forma directa de acción deletérea se relaciona con la exacerbación de la isquemia aguda observada por Wellenius y $\mathrm{col}^{16,17}$, aunque no puede descartarse una alteración de la respuesta vascular autonómica ${ }^{26}$.

B. Inflamación. La instilación o inhalación de partículas en alta concentración provocadas en animales de experimentación, determinan inflamación y daño epitelial en el árbol respiratorio ${ }^{1}$. Sin embargo, ha sido necesario disponer de mayores evidencias respecto a la inflamación que pueden provocar partículas de menor tamaño y concentración, y más aún, si esa inflama- ción puede inducir efectos sistémicos que lleven a un daño del SC-V.

Una de las mejores evidencias es la del estudio de la sICAM-1 (molécula 1 de adhesión intracelular soluble), que es un marcador de inflamación que se expresa en los leucocitos y células endoteliales. En ese estudio, se sometió a sujetos normales a un ejercicio intermitente de 2 $\mathrm{h}$ con exposición a PM en concentraciones entre 10 a $25 \mathrm{ug} / \mathrm{m}^{3}$. Se observó la aparición significativa de sICAM-1, molécula que juega un importante rol en el reclutamiento de monocitos en las lesiones ateroescleróticas y en las de la vía aérea ${ }^{27,28}$. Además, en el estudio epidemiológico de Ridker ${ }^{29}$, en sujetos aparentemente normales, los niveles plasmáticos de sICAM-1 fueron predictores de riesgo de infarto miocárdico.

Se conoce además, que el endotelio vascular es sensible a la inflamación determinada por diversas injurias (mecánicas, isquémicas e infecciosas) por acción de citoquinas, como la interleuquina 6 y la proteína $C$ reactiva, que inducen la estimulación y acumulación de monocitos y leucocitos, sea por intermediación del péptido 1 quimiotáxico para monocitos o del factor estimulante de macrófagos, que son liberados en la interfase endotelio-sangre ${ }^{30,31}$. 
C. Infección. Los estudios epidemiológicos han demostrado que la infección, específicamente la neumonía, contribuye al aumento de la mortalidad en los sujetos expuestos al $\mathrm{PM}^{3}$ y sugerían también que el material particulado podría actuar como un factor inmunosupresivo capaz de alterar la respuesta inmune normal en el huésped. Así, se ha podido demostrar que ratas infectadas con bacterias y expuestas a concentraciones entre $65 \mathrm{y}$ $150 \mathrm{ug} / \mathrm{m}^{3}$ de $\mathrm{PM}$, alteraban su respuesta inmune pulmonar y sistémica, exacerbándose el proceso infeccioso en forma significativa ${ }^{32}$. Los mecanismos involucrados parecen ser un aumento del número de bacterias, acumulación de leucocitos y aumento de las citoquinas inflamatorias, interleukinas 1 y $6^{18}$.

Se ha demostrado además que las partículas producidas por la combustión de los motores Diesel pueden inducir apoptosis de los macrófagos a nivel pulmonar, lo que lleva a una reducción de las defensas fagocíticas frente a la agresión bacteriana ${ }^{33,34}$.

D. Radicales libres o elementos ROS. Los procesos biológicos que llevan a la producción endógena de ROS son la peroxidación lipídica y la explosión respiratoria de los macrófagos. Los radicales libres reaccionan con los fosfolípidos de la membrana celular y con los ácidos grasos saturados, produciendo peroxidación con formación de radicales 〈epoxi», que inducen aumento de la permeabilidad de las membranas celulares $^{31}$. Se puede producir así un daño del endotelio vascular, que desencadena la cascada de cambios que llevan a la formación de la placa ateroesclerótica ${ }^{35,36}$.

Los elementos reactivos del $\mathrm{O}_{2}$ (ROS) pueden ser generados por el PM, y a nivel pulmonar determinan inflamación en el epitelio pulmonar y estimulación de los macrófagos. En un estudio en ratas, se midió la respuesta inmune (por medio de las inmunoglobulinas $\operatorname{IgE}$ e IgD1) en dos grupos de animales, uno tratado con antioxidantes (N-acetil-cisteína) y el otro, control. Ambos grupos fueron expuestos a concentraciones elevadas de partículas de combustión del Diesel, por 20 min durante 10 días, lo que aumentó la respuesta inmune en los no tratados con antioxidante ${ }^{37}$.
En relación al SC-V, se ha estudiado la vasodilatación inducida por NO en ratas expuestas a PM urbano. Se ha observado que la 9,10 fenantraquinona, un potente inhibidor de la NO sintetasa y otros compuestos productores de radicales libres, actúan suprimiendo la acción relajadora de la aorta y aumentando la presión arterial en ratas expuestas a $\mathrm{PM}^{1}$. Es perfectamente conocido que la acción vasodilatadora del NO es básica en la normalidad de la función del endotelio vascular 35 .

Además, en el hombre, se está estudiando el rol del estrés oxidativo en la inflamación de la vía área pulmonar sometida a PM concentrado, midiendo el contenido de $\mathrm{NO}$ y $\mathrm{CO}$ en el aire expirado, en conjunto con la medición de citoquinas en el aire espirado y en la sangre. Se espera el resultado de esos estudios ${ }^{1}$.

\section{COMENTARIO FINAL}

Es preciso reconocer que el estado de los conocimientos respecto a los efectos patológicos del PM atmosférico sobre el organismo humano y sobre el sistema pulmonar y $\mathrm{C}-\mathrm{V}$ en particular, son aún incompletos y discutibles. Uno de los aspectos problema se refiere al rol prominente o secundario que corresponde a los gases presentes en el aire contaminado de la atmósfera, como el ozono, $\mathrm{SO}_{2}, \mathrm{NO}_{2}, \mathrm{CO}_{2}$ y otros compuestos volátiles ${ }^{1}$. Otro aspecto controvertido es la validez de los estudios epidemiológicos en relación a los estudios de poblaciones bien caracterizadas y expuestas a PM definido. Ya hemos mencionado las diferencias observadas entre PM grande $0 \mathrm{PM}_{10} \mathrm{y}$ material fino $\mathrm{MP}^{2,55,9}$.

Además las técnicas que estudian la exposición al PM en el laboratorio, que sustituyen a la contaminación atmosférica real, pueden dar resultados diferentes al no considerar el tamaño y la composición química del $\mathrm{PM}^{1}$. Por ello, es posible que para los efectos reales se requiera estudiar mezclas de contaminantes gaseosos con el PM.

Además es preciso reconocer que no se debería extrapolar simplemente los hallazgos de la contaminación atmosférica a los de la contaminación intradomiciliaria. Esta puede ser tam- 
bién un importante factor de riesgo, como lo han observado Cáceres y col${ }^{38}$, en los hogares de nivel socioeconómico bajo del gran Santiago. Encontraron altas concentraciones de $\mathrm{PM}_{10}$, de $\mathrm{CO}, \mathrm{SO}_{2}$ y de hidrocarburos aromáticos absorbidos en partículas finas $\mathrm{PM}_{5}$, debido al uso de carbón y leña como calefacción, asociado a una mala ventilación domiciliaria y a una alta humedad del ambiente.

De lo anterior se desprende que la medición de los contaminantes ambientales no puede sustituir a la que corresponde a exposición personal.

\section{REFERENCIAS}

1. Epa's Science Advisory Board. The EPA's particulate Matter (PM). Health effects research centers program. Report issued. Jan 2002.

2. ZanobetTi A, Schartz J, Dockery DW. Airborne particles are a risk factor for hospital admissions for heart and lung diseases. Envir Health Perspect 2000; 108: 1071-7.

3. SchWARTZ J. Harvesting and long term exposure effects in the relation between air pollution and mortality. Am J Epidemiol 2000; 151: 440-8.

4. Pope CA, Thun MJ, Namboodiri MM, Dockery DW, Evans JS, Speizer FE et al. Particulate air pollution as a predictor of mortality in prospective study of US adults. Am J Respir Crit Care Med 1995; 151: 669-74.

5. Aranda C, Romero H. Topo-climatología de la cuenca de Santiago y sus efectos en la contaminación atmosférica y en la salud. Enf Resp Cir Tor 1989; 5: 24-30.

6. ARANDa C, AstudiLo P. Correlación entre contaminación por PM10 y mortalidad por neumonía en niños de Santiago. Rev Ch Enf Respir 1991; 7 (S): 33.

7. Ostro BD, Eskeland GS, Sánchez JM, Feyzioglu T. Air pollution and health effects: a study of medical visits among children in Santiago, Chile. Environ Health Perspect 1999; 107: 69-73.

8. Sanhueza P, Vargas C, Jiménez J. Mortalidad diaria en Santiago y su relación con la contaminación del aire. Rev Méd Chile 1999; 127: 235-42.

9. Avendaño L, Céspedes A, Stecher X, Palomina MA. Influencia de virus respiratorios, frío y contaminación aérea en la infección respiratoria aguda baja del lactante. Rev Méd Chile 1999; 127: 1073-8.
Otro elemento a considerar es que varios estudios apuntan hacia una mayor sensibilidad de pacientes de edad y con patologías crónicas previas, tanto respiratorias como cardiovasculares ${ }^{1}$.

En resumen, si bien numerosos estudios experimentales y en el ser humano demuestran que el PM atmosférico puede determinar daño orgánico, tanto pulmonar como C-V y muy probablemente en otros parénquimas, es necesario reconocer que se necesitan nuevos estudios, especialmente con centros centinelas, para validar y reforzar las observaciones que hemos analizado.

10. ZanobetTi A, Schwartz J. Are diabetics more susceptible to the health effects of airborne particles? Am J Resp Crit Care Med 2001; 164: 831-3.

11. Bateson TF, Schwartz J. Who is sensitive to the effects of particles on mortality? A case-crossover analysis. Epidemiology 2001; 12: 448.

12. Zanobetti A, Schwartz J, Gold DR. Are the sensitive subgroups for the health effects of airborne particles? Envir Health Perspect 2000; 108: 841-5.

13. Clarke RW, Coull Ba, Reinish U, Catalano PJ, KILINGsworth CR, Koutrakis P ET AL. Inhaled concentrated ambient particles are associated with hematologic and broncoalveolar lavage changes in canines. Environ Health Perspect 2000; 108: 1179-87.

14. Lutchel D, Fu K, Ghatpande P. A mouse model to study toxicity of particulate matter (PM). Am J Respir Crit Care Med 2002; 165: A301.

15. NADZIEJKO C, FANG K, GoRDOn T, CHEN LC. Quantitative analysis of arrhytmias associated with air pollution. Abstract. Workshop on cardiovascular effects associated with air pollution. Rochester NY, 2001.

16. WeuenIus G. Inhalation of concentrated ambient air particles exacerbates myocardial ischemia in conscius dogs. Env Health Perspect 2003; 3: 402-8.

17. Weunenius G, Loventt EG, Verrier RL, Coull BA, Catalano P, Koutrakis P et al. Exposure to concentrated air particles potentiates ischemic electrocardiographic changes during acute myocardial ischemia. Circulation (in press).

18. FRAMPTON MW. Systemic and cardiovascular effects of airway injury and inflammation. Ultrafine particle exposure in humans. Environ Health Perspect 2001; 109 (Suppl-4): 529-32. 
19. Zareba W, Couderc JP, Nomura A, Frampton M, Utell MJ, Peters A et al. Cardiac effects of air pollution cwhat to measure in ECG? Environ Health Perspect 2001; 109 (Suppl-4): 533-8.

20. Sulvin J, Schilderut J, Budge M. Association between short term exposure to fine particulate matter an heart rate variability in older subjects with and without heart disease. ATS 2002. Am J Resp Crit Care Med 2002; 165: A304.

21. SuLvin J. Exposure to ambient fine particulate matter and primary cardiac arrest among persons with and without clinically recognized heart disease. Am J Epidemiol 2003; 157: 501-9.

22. Zanobetti A, Schwartz J, Dockery DW. Airborne particles are a risk factor for hospital admissions for heart and lung disease. Environ Health Perspect 2000; 108: 1071-7.

23. Holguín F, Teuez-Rojo MM, Cortez M, Chow JC, Watson JG, Mannino D et al. Air pollution and heart rate variability among the elderly in Mexico City. Epidemiol 2003; 14: 521-7.

24. TANNE JH. Air pollution during warm weather increases risk of stroke. BMJ 2003; 327: 887 (Ed).

25. Cheng TJ, Hwang JS, Wang PY, Tsai CF, Chen CY, LIN SH ET AL. Effect of concentrated ambient particles on heart rate and blood pressure in pulmonary hypertensive rats. Environ Health Perspect 2003; 111: 147-50.

26. Mancia G, Grassi G, Giannattasio C, Seravalue G. Sympathetic activation in the pathogenesis of hypertension and progression of organ damage. Hypertension 1999; 34: 724-8.

27. Frampton MW, Azadniv M, Chalupa D, Morrows PE, GibB FR, OBERDOSTER G ET aL. Blood leukocyte expression of LFA-1 and ICAM-1 after inhalation of ultrafine carbon particles. Am J Resp Crit Care Med 2001; 163: A264.

28. Daigle CC, Speers DM, Chalupa D, Stewart JC, Frasier LM, AzadnIV M et al. Ultrafine particle exposure alters expression of CD40 ligand (CD154) in healthy subjects and subjects with asthma. Am J Resp Crit Care Med 2002; 165: A214.

29. Ridker PM, HenNekens CH, RoItMAn-Johnson B, Stampfer MJ, Auen J. Plasma concentration of soluble intercellular adhesion molecule and risk of future myocardial infarction in apparently healthy men. Lancet 1998; 351: 88-92.
30. Kawai $\mathrm{Y}$, Matsumoto $\mathrm{Y}$, Watanabe $\mathrm{K}$, Yamamoto $\mathrm{H}$, Sato HK, Murata M et al. Hemodynamic forces modulate the effects of cytokines on fibrinolytic activity of endothelial cells. Blood 1996; 87: 231421.

31. Van Hinsbergh VWM, Van Neuw GP, Draijer R. Regulation of the permeability of human endothelial cells monolayers. In: Vascular Endothelium. Born \& Schwartz ed. Schattahuer Ed. Berlin 1997; 61-76.

32. Zelikoff JT, Nadziejko C, Fangk, Gordon T, PremDASS C, COHEN M. Short term, low dose inhalation of ambient particulate matter exacerbates ongoing pneumococcal infections in Streptococcus pneumoniae infected rats. In: Proceedings of the Third Colloquium on Particulate Air Pollution and Human Health. Phalen R \& Berll Y, Eds. 2001; 8: 94-101.

33. Elder ACP, Gelein R, Finkelstein FN, Cox C, OBERDOSTER G. The pulmonary inflammatory response to inhaled particles is modified by age, ozone and bacterial toxin. Inhal Toxicol 2000; 12: 227-46.

34. L N, Kim S, Wong M, Froines J, Siontas C, Mil AE. Use of a stratified oxidative stress model to study the biological effects of ambient concentrated and diesel exhaust particulate matter. Inhal Toxicol 2002; 14: 459-86.

35. COOKE JP, TSAO PS. Endothelial alterations in atherosclerosis. The role of nitric oxide. In: Endothelial Function in Hypertension. SpringVerlag. Berlin 1997; 29-38.

36. Díaz MN, Frei B, Vita JA, Keaney JF. Antioxidants and atheroesclerotic heart disease. N Engl J Med 1997; 337: 408-16.

37. Whitekus MJ, Li N, Zhang M, Wang M, Horwitz M, Nelson SK ET aL. Thiol antioxidants inhibit the adjuvant effects of aerosolized diesel exhaust particles in a murine model for ovoalbumin sensitization. J Inmunol 2002; 168:2560-7.

38. Cáceres D, Adonis M, Retamal C, Ancic P, Valencia M, Ramos X ET AL. Contaminación intradomiciliaria en un sector de extrema pobreza de la comuna de La Pintana. Rev Méd Chile 2001; 129: 33-42. 